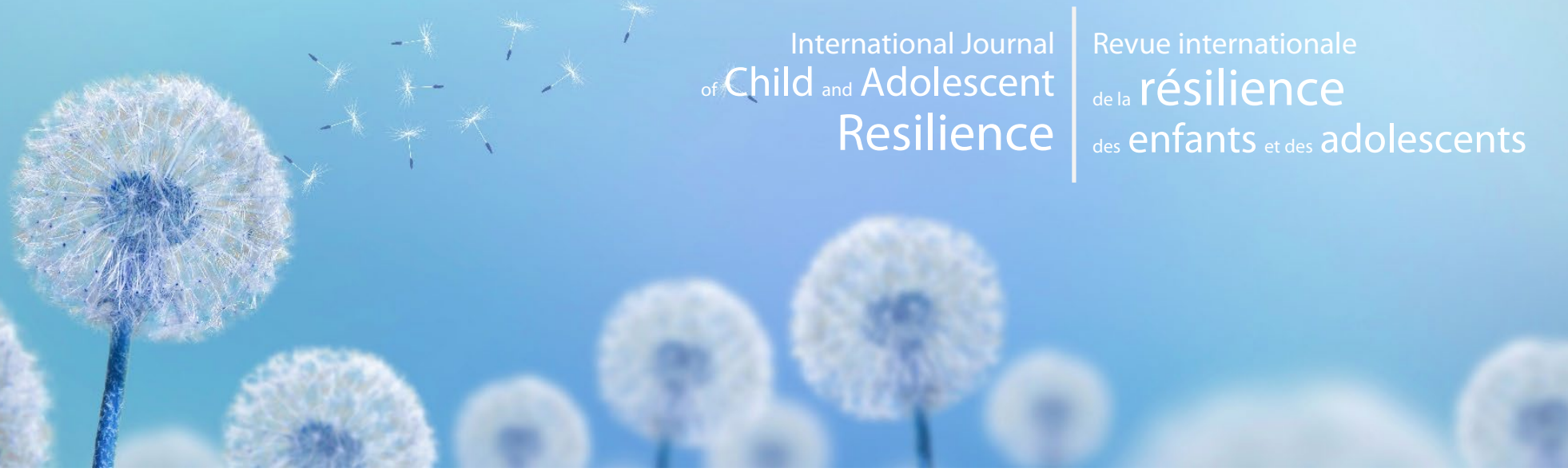

\title{
Est-ce mieux de rechercher ou de recevoir? Un modèle de soutien social à double facteur
}

\section{Sherry HAMBY ${ }^{1}$, Elizabeth TAYLOR ${ }^{1}$, Kimberly MITCHELL ${ }^{2}$, Lisa JONES ${ }^{2}$, and Chris NEWLIN ${ }^{3}$}

\footnotetext{
Life Paths Research Center and Dept of Psychology, University of the South, Sewanee, Tennessee

2 University of New Hampshire, Durham, New Hampshire

3 National Children's Advocacy Center, Huntsville, Alabama
}

Correspondance: Sherry Hamby, Ph.D., Life Paths Research Center and University of the South, PO Box 187, Sewanee, TN 37375. Courriel : sherry.hamby@sewanee.edu.

\section{Résumé}

Objectifs : Cette étude utilise une approche à double facteur pour examiner l'association entre la recherche de soutien social et le soutien reçu avec six indicateurs de fonctionnement global et 14 indicateurs de forces psychosociales.

Méthodologie: Un questionnaire mesurant les forces, le fonctionnement et la victimisation a été rempli par 440 participants âgés de 10 à 21 ans $(M=16,38, E T=3,04)$. Les participants ont été classés en quatre groupes : Interconnecté (scores élevés de recherche et de soutien social reçu; 33 \% de l'échantillon), Repoussé (scores élevés de recherche et scores bas de soutien social reçu; $12 \%$ ), Pris en charge (scores élevés de soutien social reçu, scores bas de recherche) et Isolé(scores bas de recherche et de soutien social reçu; $39 \%)$.

Résultats : En contrôlant pour l'âge, le sexe et la victimisation, le groupe de soutien social était associé aux capacités de création de sens, aux forces interpersonnelles et d'autorégulation, ainsi qu'avec chacun des indicateurs de fonctionnement, sauf les symptômes de trauma. Le groupe Isoléa obtenu des scores plus bas sur chacune des échelles de mesure et le groupe Interconnecté a obtenu les plus hauts scores sur 19 des 20 échelles. Les profils mixtes ont obtenu des scores entre ces deux extrêmes. Notamment, le groupe Repousséa rapporté des scores plus élevés pour plusieurs forces ainsi que le bien-être spirituel non-théiste que le groupe Pris en charge. Les scores du groupe Pris en charge n'étaient jamais significativement plus élevés que ceux du groupe Repoussé.

Implications: Les compétences individuelles et les attitudes favorables à la recherche d'aide pourraient avoir plus d'impact que le soutien social fourni par les autres. Les participants du groupe Repoussé pourraient exploiter d'autres forces quand l'environnement social est non favorable.

Mots-clés : Soutien social, résilience, écologie sociale, jeunes, recherche de soutien social, soutien social reçu. 


\section{Introduction}

Le soutien social serait un facteur de protection important dans les études portant sur la victimisation, la résilience et plusieurs autres phénomènes similaires à l'enfance et l'adolescence (Chu et al., 2010). Dans plusieurs études, le soutien social serait un indicateur clé de l'écologie sociale ou du contexte psychosocial plus général dans lequel les individus s'adaptent à l'adversité. Un meilleur soutien social serait associé à un plus grand bien-être et à un plus grand ajustement psychosocial (Chu et al., 2010). Les recherches sur le soutien social s'appuient généralement sur des mesures de cotation globale du soutien social perçu des individus (p. ex., Clara et al., 2003; Zimet et al., 1988). Bien que ces recherches aient été utiles pour identifier l'importance du soutien social dans l'écologie sociale, des mesures aussi globales laissent sans réponse des questions qui auraient pu guider les interventions cliniques. Par exemple, ces ressources doivent être obtenues d'une certaine façon. La recherche de soutien social est souvent étudiée séparément de la quantité de soutien social disponible ou offerte, mais elles sont logiquement reliées et comprendre ces connexions est important théoriquement et pour les interventions (Kim et al., 2008). L'objectif de cette étude est d'explorer si le modèle de soutien social à double facteur révèle des schémas où la recherche et la réception de soutien social sont étroitement associées avec des forces psychosociales et des indicateurs de bien-être au sein d'un échantillon de jeunes provenant du sud des États-Unis.

\section{Soutien social et écologie sociale}

L'écologie sociale comprend les familles, les réseaux de pairs, les communautés et les sociétés qui contribuent au fonctionnement d'un individu (Bronfenbrenner, 1979; Kelly, 1968; Trickett et al., 1983). Ces éléments du réseau social peuvent apporter des ressources extérieures aux jeunes, par exemple par le biais de ressources communautaires comme des cliniques et des groupes de soutien social (Grych et al., 2015). Bien que l'écologie sociale des jeunes contienne plusieurs éléments, comme l'efficacité collective ou l'implication parentale (Fritz, de Graaff, Caisley, Van Harmelen \& Wilkinson, 2018; Sampson, Raudenbush \& Earls, 1997), le soutien social est l'un des facteurs les plus étudiés. Le soutien social est souvent défini comme étant la mise à disposition de ressources matérielles et immatérielles (c.-àd., psychologiques) dans le but d'aider une personne, surtout en période de stress (Chu et al., 2010). La plupart des études sur le soutien social se sont concentrées sur les personnes de l'environnement social immédiat, en particulier les membres de la famille, les pairs et, dans le cas des jeunes, les adultes significatifs tels que les enseignants, les entraîneurs ou les dirigeants de groupes communautaires (Chu et al., 2010; Turner et al., 2017; Zimet et al., 1988).

La plupart des mesures de soutien social existantes sont axées sur l'évaluation de ces ressources externes, en se concentrant soit sur le nombre de personnes présentes dans le réseau social d'un individu ou l'aide qu'elles peuvent apporter, soit sur les ressources spécifiques auxquelles une personne peut accéder. Une des mesures les plus utilisées est le Multidimensional Scale of Perceived Social Support (MSPSS; Zimet et al., 1988) qui évalue le soutien social perçu provenant de trois groupes de personnes clés : les membres de la famille, les amis et les autres personnes importantes. Bien que cet instrument de mesure ait été développé auprès d'adultes, l'échelle MSPSS est souvent utilisée avec des adolescents (p. ex., Frison \& Eggermont, 2015) et a aussi été adaptée à l'évaluation du soutien provenant des figures non-parentales significatives des jeunes (Turner et al., 2017). D'autres outils populaires permettent d'évaluer le soutien social en demandant aux répondants d'indiquer le nombre de personnes qui pourraient fournir certains types de soutien ainsi que leur satisfaction à l'égard du soutien reçu (Sarason et al., 1983) ou en déterminant l'accès au « soutien disponible en cas de besoin » (Sherbourne \& Stewart, 1991). Toutefois, d'un point de vue écologique, ces mesures des ressources externes n'évaluent qu'un côté de la transaction interpersonnelle.

\section{La recherche de soutien social}

Comme tous les éléments de l'écologie sociale, le soutien social se base sur une interaction entre l'individu et son contexte social (Chan et al., 2016). Dans un scénario optimal, une personne en détresse demande de l'aide, puis les membres de son réseau social se rallient autour d'elle et lui fournissent les ressources matérielles et immatérielles demandées. Demander de l'aide aux amis et aux proches après une expérience traumatisante n'est pas rare (contrairement à ce qui est véhiculé par certains stéréotypes sur les victimes passives), comme rapporté par la majorité des participants de différentes études (p. ex., Barrett \& Pierre, 2011; Sullivan et al., 2010). La recherche d'aide est aussi fréquente chez les jeunes (Bundock et al., 2020). Le modèle rechercher-et-recevoir est implicite dans de nombreux programmes de prévention et d'intervention qui tentent d'accroître la recherche d'aide par opposition à l'augmentation directe du soutien social. Par exemple, l'encouragement à la recherche d'aide est l'élément le plus courant dans les programmes de prévention de la violence chez les jeunes aux États-Unis, et est inclus dans $88 \%$ des programmes (Finkelhor et al., 2014). En revanche, seulement un programme sur cinq essaie directement d'améliorer la 
communication entre les jeunes et les parents (Finkelhor et al., 2014), ce qui peut améliorer la recherche d'aide en aidant les jeunes à se sentir plus à l'aise de se confier à leurs parents et ces derniers à se sentir davantage en mesure de réagir à ces dévoilements. Cependant, certaines recherches montrent que ce modèle idéalisé - où l'individu recherche un soutien qui lui est ensuite fourni - ne se produit pas toujours (p. ex., Foynes \& Freyd, 2011).

Bien que de nombreux programmes de prévention et d'intervention recommandent sans équivoque la recherche d'aide et supposent implicitement que toute recherche d'aide sera satisfaite par des réponses utiles, il existe des preuves considérables que ce n'est pas toujours le cas. Le « trauma induit par le système », soit un type de trauma secondaire dans lequel les réponses peu utiles des professionnels des services sociaux exacerbent le problème d'une victime, est malheureusement courant (Conners-Burrow et al., 2013). Dans le domaine de la violence sexuelle, les réponses peu utiles, voire même traumatisantes, des professionnels sont si courantes que les réponses des forces de l'ordre et de la communauté sont parfois appelées «le second viol » (Campbell et al., 2001). La recherche sur les dévoilements aux pairs et aux proches révèle également l'occurrence de nombreuses réponses peu aidantes, comme le rejet, la stigmatisation et le blâme des victimes (Foynes \& Freyd, 2011). Une grande partie de ces recherches a été effectuée auprès de victimes adultes, mais une étude menée dans une école secondaire belge a révélé que les élèves qui recherchaient du soutien social sur Facebook et qui n'en percevaient pas déclaraient être d'humeur plus dépressive que les autres jeunes (Frison \& Eggermont, 2015). II est aussi possible que les bénéficiaires des demandes d'aide ne soient pas en mesure de fournir l'aide demandée. Ces écarts par rapport au modèle idéal suggèrent qu'il faut accorder plus d'attention aux modèles de recherche et de réception de soutien social, et mieux reconnaître que la recherche de soutien social ne réussit pas toujours.

\section{Un modèle de soutien social à double facteur}

Bien que la réception et la recherche de soutien social aient été largement étudiées, peu de recherches ont exploré les interconnexions de ces deux aspects du soutien social (Kim et al., 2008). Mis à part le nombre relativement restreint de recherches sur les traumatismes induits par le système, la plupart des recherches supposent que les deux phénomènes, la recherche et la réception, sont étroitement liés. Dans la présente étude, nous suivons les principes d'abord élaborés pour le modèle à double facteur de la santé mentale. Le modèle à double facteur de la santé mentale a montré que deux indicateurs du fonctionnement psychologique couramment étudiés, la psychopathologie et le bien-être, ne devraient pas être considérés comme de simples pôles opposés d'un même continuum. Au contraire, les individus peuvent avoir un niveau de bien-être élevé malgré des niveaux importants de symptômes psychologiques, ou avoir peu de symptômes psychopathologiques et quand même avoir un niveau bas de bien-être (Greenspoon \& Saklofske, 2001; Suldo \& Shaffer, 2008). Ces résultats ont contribué à une compréhension plus nuancée de la santé mentale, car il est maintenant reconnu que les personnes correspondant à ces profils mixtes peuvent être distinguées de celles ayant des scores uniformes pour les deux indicateurs (Antaramian et al., 2010; Greenspoon \& Saklofske, 2001; Suldo \& Shaffer, 2008).

Nous proposons un modèle similaire de soutien social à double facteur. Ce modèle reconnaît que le fait de recevoir un niveau élevé de soutien social est souvent dû à un niveau élevé de recherche de soutien, c'est-à-dire un modèle qui reflète des niveaux élevés d'interconnexion entre un jeune et son environnement social. Inversement, le fait de recevoir un faible niveau de soutien peut être dû à des efforts minimes pour obtenir de l'aide en cas de besoin, ce qui reflète l'isolement social en période de détresse. Cependant, rechercher et recevoir sont deux actions conceptuellement distinctes et, par conséquent, deux profils mixtes peuvent également émerger. Un profil mixte peut être constitué de personnes qui recherchent du soutien social, mais qui n'en reçoivent pas: le groupe Repoussé. Comme mentionné, ce groupe a fait l'objet de certaines études, bien qu'elles portent principalement sur les réponses ouvertement négatives et nuisibles (e.g., Campbell et al., 2001; Foynes \& Freyd, 2011; Frison \& Eggermont, 2015). Certains membres de ce groupe peuvent recevoir une aide minimale ou ne pas recevoir de réponse du tout. Bien qu'une grande partie de la recherche sur les réponses négatives à la recherche d'aide ait été menée auprès d'adultes, ce profil semble potentiellement problématique pour les jeunes, qui auront encore moins de capacité que la plupart des adultes à accéder aux ressources nécessaires par eux-mêmes. Le quatrième profil n'a fait l'objet de peu, voire aucune étude. II s'agit de personnes pour lesquelles un soutien social considérable est offert, malgré le peu d'efforts déployés pour le solliciter. Ce profil pourrait être particulièrement important pour les jeunes dont les personnes qui prennent soin d'eux essaient de répondre à leurs besoins, peu importe s'il est exprimé de façon efficace ou non. Nous appelons ce sous-groupe Pris en charge.

Examinées à travers le modèle de soutien social à double facteur, de nombreuses mesures existantes de soutien social produisent des scores ambigus. Par exemple, dans le MSPSS (Zimet et al., 1988), l'une des mesures de 
soutien social les plus couramment utilisées, un score faible pourrait indiquer un problème de réponses inefficaces de la part du réseau social, mais il pourrait aussi indiquer un problème dans la recherche d'aide. Aussi, un score élevé sur l'échelle n'indique pas si le soutien a été sollicité ou simplement offert. D'autres mesures présentent des problèmes similaires, par exemple la documentation des ressources connues et disponibles (comme dans Sherbourne \& Stewart, 1991) pourrait suggérer un manque de connaissances, une faible recherche d'aide, une communauté offrant peu de ressources ou la combinaison de plusieurs de ces facteurs. D'autres mesures, comme le Child and Adolescent Social Support Scale (Malecki \& Demaray, 2002), se concentrent aussi sur les ressources disponibles, mais n'évaluent pas nécessairement la recherche d'aide et n'indiquent pas clairement si le soutien est offert suite à une demande d'aide ou si ce sont simplement des ressources relationnelles généralement disponibles. De plus, certains éléments, comme lorsque les parents «soulignent poliment mes erreurs», peuvent ne pas être perçus comme du soutien. Pour ce qui est d'orienter la prévention et l'intervention, les résultats des recherches ne peuvent pas indiquer, avec de telles mesures, s'il est plus important que les gens demandent de l'aide ou qu'ils reçoivent un soutien externe.

Étant donné le manque relatif de connaissances sur les façons dont le soutien social recherché et reçu peuvent interagir, il est nécessaire d'approfondir les recherches sur la relation entre ces phénomènes, et ce, surtout chez les jeunes. Pour aider à comprendre les façons dont ces quatre groupes - Interconnecté, Isolé, Repoussé et Pris en charge - fonctionnent dans l'écologie sociale plus générale et comment ils peuvent contribuer à la résilience, il est nécessaire d'associer ces profils à d'autres facteurs de protection et à d'autres indicateurs du fonctionnement. Le Resilience Portfolio Mode/ (Grych et al., 2015; Hamby, Grych et al., 2018) classe les forces psychosociales en trois domaines : la régulation (gestion des émotions et des comportements), la création de sens (connexion avec quelque chose de plus grand que soi) et les relations interpersonnelles (relations avec l'écologie sociale plus large). Le Resilience Portfolio Mode/ souligne également la nécessité de mesurer une gamme de conséquences possibles, y compris le bien-être psychologique, physique et spirituel.

\section{La présente étude}

La présente étude a examiné la recherche et la réception de soutien social dans un échantillon de jeunes provenant du sud des États-Unis. Ces jeunes ont été classés en quatre groupes : Interconnecté(recherche et obtention de soutien social élevé), Repoussé (recherche élevée, obtention faible), Pris en charge (recherche faible, obtention élevée) et Isolé (recherche et obtention faible) afin de déterminer si ces groupes se distinguent sur le plan de 14 forces psychosociales et 6 mesures de fonctionnement psychologique, physique et spirituel après avoir contrôlé pour la victimisation, l'âge et le sexe. Nous avons prédit que le fait d'avoir des scores élevés aux deux types de soutien social (Interconnecté) serait associé à des scores plus élevés sur les échelles de fonctionnement et de forces psychosociales, conformément aux recherches antérieures et au Resilience Portfolio Model, alors qu'obtenir des scores bas aux deux types de soutien (Isolé) serait associé à des scores plus faibles aux indicateurs de forces et de bien-être. Étant donné la rareté des recherches disponibles sur l'impact de la recherche versus l'obtention de soutien social, nous avons exploré les associations des deux profils mixtes (Repoussé et Pris en charge) à l'aide de nos indicateurs de forces et de bienêtre.

\section{Méthodologie}

\section{Participants}

L'échantillon est composé de 440 jeunes provenant de quatre États du sud des États-Unis (AL, GA, MS, TN). Les participants étaient âgés de 10 à 21 ans $(M=16,38, E T=3,04)$, dont 61,1\% étaient des filles. En ce qui concerne la race et l'identité ethnique, les participants se sont identifiés comme étant $69,9 \%$ blancs ou Européens américains (nonlatinos), 17,1\% noirs ou afro-américains (non-latinos), 5,6\% multiraciaux, 3,9\% latinos, 1,9\% Amérindiens ou Autochtones de l'Alaska (non-latinos) et 1,6\% asiatiques (non-latinos). Plus de la moitié de l'échantillon (61\%) vivait dans une région rurale $(27,4 \%)$ ou une petite ville $(33,6 \%)$, avec une population de moins de 20000 habitants. Les autres participants ont déclaré vivre dans des villes (14,1\% dans des villes de 20000 à 100000 habitants), des grandes villes (15\% dans des villes de moins de 300000 habitants) et dans des métropoles ou des banlieues (9,9\%). Le revenu médian du ménage dans leur comté de résidence (données de 2016, les plus récentes disponibles au moment de la collecte des données) était de $47713,40 \$(E T=11635,61)$, soit $19 \%$ de moins que la moyenne de 59039 \$ pour les États-Unis. 


\section{Procédure}

Les participants ont été recrutés dans des organismes jeunesse en 2017 et 2018. Les organismes jeunesse ont été recrutées dans la communauté environnante en assistant à des réunions de conseils de santé locaux (organisations de comté des organismes sans but lucratif et de services de la région) et par le bouche-à-oreille. Si un organisme souhaitait participer, il nous contactait par courriel ou par téléphone pour convenir d'une heure et d'un lieu pour répondre au sondage, généralement lors d'une de ses réunions régulières. Nous avons recueilli des données auprès de quatorze organismes jeunesse. Conformément aux exigences de notre organisme de financement, des allocations ont été offertes aux organismes jeunesse, et non aux particuliers, afin de soutenir leurs programmes. Les organismes ont reçu une allocation de 20 \$ par participant. Le sondage a été répondu sous forme d'entrevue assistée par ordinateur, à l'aide de la plate-forme logicielle SNAP11 sur des tablettes électroniques. En moyenne, il a fallu environ 22 minutes pour répondre au sondage. Le consentement éclairé, y compris le consentement parental pour les mineurs, a été obtenu pour tous les participants. Les critères d'inclusion comprenaient le fait d'être âgé de 10 à 21 ans, d'avoir obtenu le consentement parental pour les moins de 18 ans et de pouvoir répondre au sondage en anglais. Toutes les procédures ont été approuvées par le comité institutionnel d'éthique de la recherche. Le taux de complétion global était de $92 \%$, ce qui est un excellent résultat selon les normes d'enquête actuelles, certains taux de complétion étant souvent inférieurs à $70 \%$ et parfois à $50 \%$ (Abt SRBI, 2012; Galesic \& Bosnjak, 2009).

\section{Mesures}

Développement et validation des mesures. Les mesures ont été conceptualisées, perfectionnées et validées en utilisant une procédure à méthodes mixtes (Hamby, Taylor, et al., 2018). L'un des objectifs de ce projet de plus grande envergure était d'élaborer et de valider de brèves mesures des forces et du bien-être pouvant être utilisées auprès des jeunes. La validité des instruments a été établie à l'aide de corrélations modérées avec des construits connexes et était conforme aux travaux antérieurs sur les mesures du Resilience Portfolio (Hamby, Grych, et al., 2018). Sauf indication contraire, les catégories de réponse correspondaient une échelle de Likert en 4 points, 1 signifiant « Pas vrai pour moi » et 4 signifiant «En grande partie vrai pour moi ». Les données manquantes (intervalle de 1 à 3,2 \%, moyenne de 1,1\%) ont été imputées en fonction des réponses à d'autres items de la même échelle. Dans tous les cas, les scores élevés représentent des niveaux plus élevés du construit. De plus amples détails sur chaque mesure sont présentés ci-dessous.

Le soutien social a été mesuré à l'aide de 11 items fondés sur les données recueillies pendant la phase qualitative de cette étude (Hamby, Taylor, et al., 2018). Nous avons effectué une analyse factorielle exploratoire sur ces 11 items, en utilisant la factorisation en axes principaux comme méthode d'extraction. Deux facteurs ont été extraits. Le premier, que nous avons appelé Soutien social recherché, expliquait 46,7 \% de la variance, avait une valeur propre (eigenvalue) de 5,14 et comprenait six items dont la saturation se situait au-dessus du seuil de 0,4. Le deuxième facteur, que nous avons appelé Soutien social reçu, expliquait 14,5\% de la variance, avait une valeur propre (eigenvalue) de 1,59 et comprenait les cinq autres items. Voir le Tableau 1 pour les items individuels et leurs saturations. L'échelle Soutien social recherchéqui en résulte $(6$ items, $a=, 89)$ évalue les efforts faits par les jeunes pour obtenir de l'aide ainsi que leurs attitudes à l'égard de la demande d'aide. L'échelle Soutien social reçu (5 items, $a=, 80$ ) évalue l'aide ou l'encouragement fourni par d'autres personnes en période de détresse. 
Tableau 1. Analyse factorielle des items de soutien social

\begin{tabular}{|c|c|c|c|}
\hline \multirow[b]{2}{*}{ Item } & \multicolumn{2}{|c|}{ Saturation du facteur } & \multirow[b]{2}{*}{$\begin{array}{c}\text { \% ayant répondu } \\
\text { «Particulièrement } \\
\text { vrai » }\end{array}$} \\
\hline & $\begin{array}{l}\text { Soutien social } \\
\text { reçu }\end{array}$ & $\begin{array}{l}\text { Soutien social } \\
\text { recherché }\end{array}$ & \\
\hline Quelqu'un m’a aidé à me changer les idées. & ,79 & & 55,2 \\
\hline Quelqu'un m’a réconforté. &, 73 & & 62,6 \\
\hline Quelqu'un était là pour moi lors de moments difficiles. &, 63 & & 64,6 \\
\hline $\begin{array}{l}\text { Quelqu'un m’a donné un endroit où je pouvais me réfugier pour un } \\
\text { moment. }\end{array}$ &, 62 & & 42,0 \\
\hline Quelqu'un est venu chercher de l'aide avec moi. &, 48 & & 33,5 \\
\hline Je me sens mieux lorsque je parle aux gens de ce qui se passe. & &, 80 & 56,6 \\
\hline $\begin{array}{l}\text { Cela m'aide de discuter de mes idées avec quelqu'un lorsque j'ai un } \\
\text { problème. }\end{array}$ & &, 76 & 56,0 \\
\hline Je parle à quelqu'un pour m'aider à résoudre des problèmes. & &, 75 & 48,8 \\
\hline Discuter avec quelqu'un m'aide lorsque je suis bouleversé.e. & &, 68 & 57,9 \\
\hline Parler à quelqu'un qui a vécu la même chose m’aide. & &, 65 & 58,3 \\
\hline Je demande aux gens de m'aider à prendre des décisions difficiles. & &, 63 & 46,0 \\
\hline Valeurs propres initiales & 5,14 & 1,59 & \\
\hline \% de la variance expliquée & 46,69 & 14,45 & \\
\hline
\end{tabular}

Ces scores ont été utilisés pour créer quatre groupes de soutien social, en utilisant une répartition médiane pour chaque échelle de soutien social (jusqu'au $50^{\mathrm{e}}$ percentile inclusivement $=0$, plus haut que le $50^{\mathrm{e}}$ percentile $=1$ ), qui intègrent les compétences et les attitudes individuelles des jeunes en matière de recherche d'aide (un atout) et le niveau de soutien extérieur offert (ressources). Le groupe Interconnectéa obtenu un score élevé tant pour la recherche que pour l'obtention de soutien social. Le groupe Pris en charge a obtenu un score élevé pour le soutien social reçu, malgré des scores faibles pour la recherche de soutien social. Le profil du groupe Repousséétait également mixte, avec une forte recherche, mais seulement une faible réception de soutien social. Enfin, le groupe Isoléa obtenu de faibles scores tant pour la recherche que pour l'obtention de soutien social. Voir le Tableau 2 pour une représentation de ces groupes et les pourcentages de chacun.

Tableau 2. Le modèle de soutien social à double facteur et les pourcentages de chaque groupe dans cet échantillon

\begin{tabular}{lcc}
\hline \multirow{2}{*}{$\begin{array}{l}\text { Soutien social reçu } \\
\text { Réception faible }\end{array}$} & Recherche faible & Soutien social recherché \\
& Recherche élevée & Repoussé \\
\cline { 2 - 3 } & $39 \%(n=172)$ & $12 \%(n=53)$ \\
Réception élevée & Pris en charge & Interconnecté \\
& $16 \%(n=69)$ & $33 \%(n=146)$ \\
\hline
\end{tabular}

Note. Total $n=440$.

Les forces d'autorégulation évaluent divers aspects du contrôle de soi, surtout lorsqu'on fait face à des difficultés. Ces échelles ont été développées ou adaptées par le biais d'une procédure de méthodes mixtes décrite cidessus (Hamby, Taylor, et al., 2018). L'échelle Endurance psychologique est une version simplifiée à 5 items d'une mesure (Hamby, Grych, et al., 2018) permettant d'évaluer la capacité d'une personne à persévérer malgré les difficultés $(a=$,69). Un exemple d'item est « Lorsque des choses difficiles arrivent, je préfère les confronter directement ». Rétablir l'affect positif $(a=, 81)$ est une mesure de 6 items qui évalue la capacité à retrouver une humeur joyeuse après avoir ressenti de la détresse. Un exemple d'un item est « Je peux me remonter le moral après une mauvaise journée ». L'échelle Autonomie mesure la capacité de s'adapter en utilisant ses propres ressources ( 3 items, $a=, 81$ ). Un exemple d'item est « J'aime régler mes problèmes par moi-même ». L'échelle Contrôle des impulsions évalue l'autorégulation comportementale (5 items, $a=, 63$ ). Un exemple d'item est « Je pense avant d'agir ».

Les capacités de création de sens évaluent les moyens par lesquels les individus cherchent à s'épanouir, souvent en se connectant à quelque chose de plus grand qu'eux (Hamby, Taylor, et al., 2018). Raison d'être (6 items; a $=, 88$ ) implique de sentir qu'il y a un sens à la vie et une raison de vivre. Adapté pour les jeunes d'une version antérieure (Hamby, Grych, et al., 2018). Un exemple d'item est : « Mes valeurs donnent un sens à ma vie ». Importance (5 items; $a$ 
$=, 86)$ évalue dans quelle mesure les participants se sentent appréciés et valorisés par les autres. Un exemple d'item est « Je me sens apprécié.e par ma famille et mes amis ». Orientation future (6 items; $a=, 79)$ mesure le désir d'amélioration personnelle. Exemple d'item: «Les choix que je fais aujourd'hui sont importants pour mon futur». Motivation relationnel/e ( 3 items; $a=, 70$ ) fait référence au sentiment d'être inspiré par des personnes importantes dans sa vie. Exemple d'item : « Je veux que les gens dans ma vie soient fiers de moi ». Sens religieux ( 6 items; $a=, 94$ ) évalue la foi et l'engagement des individus dans les pratiques religieuses/spirituelles. Cette échelle a été adaptée pour les jeunes à partir d'une version précédente (Hamby, Grych, et al., 2018). Exemple d'item : «Lorsque j'ai un problème, je demande aux autres de prier pour moi ».

Les forces interpersonnelles comprennent les compétences relationnelles des participants ainsi que des indicateurs de soutien de leur environnement social plus large. Compassion (Hamby, Grych, et al., 2018) mesure la façon dont les gens interagissent avec les autres de façon bienveillante et aidante ( 4 items, $a=80$ ). Un exemple d'item est «Si quelqu'un vit des moments difficiles, j'essaie de prendre soin de cette personne ». Soutien de la communauté (Roberts et al., 2015) est composé de six items qui évaluent le degré d'entente et d'entraide entre voisins $(a=, 80)$. Un exemple d'item est « Les gens de mon quartier offrent de s'entraider ». Les autres échelles de forces interpersonnelles ont été élaborées selon le processus mixte décrit ci-dessus et ont été conçues pour saisir d'autres aspects de l'écologie sociale des jeunes (Hamby, Taylor, et al., 2018). Connexion au groupe (6 items, a $=, 80$ ) évalue les sentiments de proximité et de soutien des groupes de pairs. Un exemple d'item est « J'ai appartenu à un groupe ou à une équipe de gens qui me défendaient ». Climat scolaire $(6$ items, $a=, 78)$ mesure les caractéristiques des climats scolaires sains, comme «Le bâtiment de mon école est en bonne condition ». Engagement des professeurs ( 5 items, $a=, 86$ ) évalue les expériences des jeunes avec des enseignants enthousiastes et bienveillants. Un exemple d'item est « J'ai eu un professeur qui voulait que je réussisse à l'école».

La polyvictimisation a été évaluée avec la version abrégée du Questionnaire de victimisation juvénile (JVQ Key Domains Short Form), qui inclut 10 items évaluant I'historique d'une variété de formes de victimisation interpersonnelle vécues tout au long de la vie, adapté du JVQ original (Finkelhor et al., 2005; Hamby, Taylor, et al., 2018).). Un exemple d'item est «Durant ton enfance, est-ce qu'un de tes parents a menacé de blesser ton autre parent, et qu'il te semblait qu'il pourrait vraiment être blessé ? ». Les items dichotomiques (« oui » ou « non ») ont été additionnés pour créer un score total de victimisation $(a=, 73)$. Le nombre médian de victimisations déclarées par les jeunes était de 3 , avec une moyenne de $M=3,40$ ( $E T=2,43$ ). Dans cet échantillon, plus de 3 jeunes sur $4(75,9 \%)$ ont déclaré deux formes de victimisation ou plus, et près de 9 jeunes sur $10(89,3 \%)$ ont déclaré au moins une forme de victimisation.

Fonctionnement global. Plusieurs indicateurs ont été utilisés pour évaluer le fonctionnement global. Dans le but d'examiner les multiples aspects du bien-être, des mesures ont été utilisées pour évaluer le bien-être et le fonctionnement psychologique, physique et spirituel. Symptômes de trauma (8 items, $a=91$ ) évalue une variété de sentiments de dysphorie, d'anxiété ou de culpabilité (Hamby, Taylor, et al., 2018). Un exemple est «Se sentir préoccupé.e ou anxieux.e dans le dernier mois ». Un score élevé indique plus de symptômes. Health-related Quality of Life (HRQOL; 5 items, $a=, 64$ ) se base sur la mesure du CDC (Centers for Disease Control and Prevention, 2000), simplifiée et adaptée le bien-être physique (Banyard et al., 2017). Un exemple d'item : « Durant le dernier mois, pendant combien de jours ta santé t'a empêché de faire tes activités habituelles, comme aller à l'école ou passer du temps avec des amis ?». Des scores élevés sur cet index indiquent une meilleure qualité de vie liée à la santé au cours du moins précédent l'étude. Bien-être subjectif(7 items, $a=, 90)$ examine la satisfaction générale de la vie fondée sur les forces, en opposition à l'absence de symptômes de psychopathologie (Hamby, Grych, et al., 2018). Un exemple d'item est « Je me sens vraiment bien par rapport à ma vie ». D'autres mesures du bien-être ont été développées en utilisant une approche basée sur les méthodes mixtes décrites précédemment (Hamby, Grych, et al., 2018). Bien-être familial(7 items, a =,90) évaluait le bien-être subjectif de la famille immédiate et des proches vivant avec l'enfant. Un exemple d'item : "Ma famille est heureuse ». Bien-être spiritue/inclus à la fois une sous-échelle théiste (5 items, $a=, 95)$ et une sous-échelle non-théiste ( 5 items, $a=82$ ). La sous-échelle théiste évalue le bien-être fondé sur la présence d'un dieu ou d'une puissance supérieure similaire. Un exemple d'item est «Je me sens mieux quand je parle à Dieu ou à une force supérieure ». La sous-échelle non-théiste illustre un sentiment similaire d'émerveillement ou de bien-être provenant de sources moins religieuses, telles que ressentir un lien avec la nature. Un exemple d'item est « J'ai l'impression que tous les êtres vivants sont connectés». 


\section{Analyses}

Pour faciliter les comparaisons entre les mesures, tous les scores des échelles ont été standardisés en scores Z (moyennes converties à 0 avec un écart type de 1). Les statistiques descriptives ont été examinées pour identifier les caractéristiques de l'échantillon. Des analyses de covariance (ANCOVAs) ont été conduites pour examiner la différence entre les quatre groupes de soutien social, Interconnecté, Repoussé, Pris en charge et Isolé, suivi d'analyse post-hoc de comparaisons de moyennes. L'âge, le sexe, et la polyvictimisation ont été inclus dans le modèle comme covariables, et les forces psychosociales et les indicateurs de fonctionnement global comme variables dépendantes.

\section{Résultats}

\section{Les expériences de soutien social}

Plusieurs formes de soutien social ont été rapportées par une proportion substantielle de jeunes de l'échantillon. L'item le plus endossé des 11 items était «Quelqu'un était là pour moi lors de moments difficiles », avec $64 \%$ de l'échantillon qui a répondu que c'était «En grande partie vrai » pour eux. L'item le moins endossé appartenait aussi à l'échelle Soutien social reçu: «Quelqu'un est venu chercher de l'aide avec moi », dont seulement 33,5\% ont répondu que c'était «En grande partie vrai » pour eux. Sur l'échelle Soutien social recherché, l'item le plus endossé est «Parler à quelqu'un qui a vécu la même chose m'aide », avec 58 \% disant que c'était « En grande partie vrai ». Quelques formes de recherche de soutien social étaient moins communes comme « Je demande aux gens de m'aider à prendre des décisions difficiles » et « Je parle à quelqu'un pour m'aider à résoudre des problèmes », où moins de la moitié des jeunes ont indiqué que c'était «En grande partie vrai » (respectivement $46 \%$ et $49 \%$ ).

\section{Quatre groupes de soutien social et autres forces}

Les ANCOVAs ont révélé plusieurs différences significatives dans les forces psychosociales parmi les quatre groupes de soutien, après avoir contrôlé pour l'âge, le sexe et la polyvictimisation.

Forces d'autorégulation. Nous avons d'abord examiné les différences entre les forces individuelles d'autorégulation des quatre groupes de soutien social : Interconnecté, Repoussé, Pris en charge et Isolé. II y avait des différences significatives pour les quatre forces d'autorégulation, $p<0,01$. Voir le Tableau 3. Pour ce qui est de l'endurance et du rétablissement de l'affect positif, les jeunes des groupes Interconnecté et Repoussé, qui étaient semblables entre eux, ont obtenu des scores plus élevés que les jeunes des groupes /soléet Pris en charge. Les groupes Isolé et Pris en charge n'étaient pas significativement différents l'un de l'autre. En ce qui concerne l'autonomie, la situation était semblable, mais le groupe Repoussé ne différait pas des deux autres. Pour le contrôle des impulsions, seul le groupe Interconnecté a rapporté un niveau de contrôle des impulsions significativement plus élevé que les autres groupes. La répartition des scores était similaire pour les quatre variables, le groupe Isolé rapportant les scores les plus bas pour les quatre forces et le groupe Pris en charge se situant à l'avant-dernier rang. Le groupe Interconnecté a obtenu les scores les plus élevés, sauf pour l'endurance, pour laquelle le groupe Repoussé rapportait des scores légèrement (mais pas significativement) plus élevés. Pour toutes les comparaisons appariées, $p<, 05$.

Les covariables étaient significatives dans certaines de ces analyses. Pour l'âge, les jeunes plus âgés ont rapporté des niveaux plus élevés d'endurance, $p<, 05$, et de contrôle des impulsions, $p<, 01$. En ce qui a trait au sexe, les jeunes de sexe masculin ont rapporté des niveaux plus élevés d'endurance, $p<, 05$, et de rétablissement de l'affect positif, $p=, 001$. Concernant la polyvictimisation, des niveaux plus bas de victimisations étaient associés à un meilleur contrôle des impulsions, $p<, 001$, et à de meilleures facultés de rétablissement de l'affect positif, $p<, 001$.

Les capacités de création de sens. En ce qui concerne les capacités de création de sens, il y avait des différences significatives entre les groupes de soutien social pour toutes les cinq forces, $p<, 001$. Voir aussi le Tableau 3. Les comparaisons des moyennes indiquent que le groupe Isolé était significativement plus bas que les trois autres groupes pour chaque force, sauf celle concernant l'orientation vers l'avenir qui démontrait la même répartition que pour l'endurance et la faculté de rétablissement de l'affect positif, c'est-à-dire que les groupes Isolé et Pris en charge avaient des scores significativement plus bas que les groupes Interconnecté et Repoussé. En ce qui concerne le sentiment de raison d'être et la création de sens religieux, les jeunes du groupe Interconnecté en ont rapporté davantage que les autres jeunes. Les jeunes du groupe Interconnecté qui ont demandé et reçu de l'aide, ont rapporté significativement plus de sens que les autres. Toutefois, pour ce qui est du sentiment d'importance et de la motivation 
relationnelle, alors que les jeunes Isolé ont rapporté les scores les plus bas, les trois autres groupes n'étaient pas significativement différents les uns des autres. Toutes les comparaisons par paires étaient significatives, $p<, 05$.

En ce qui a trait aux covariables, l'âge était significatif pour l'orientation vers l'avenir, $p<, 05$, et pour la création de sens religieux, $p<, 01$, indiquant que les participants s'orientaient davantage vers l'avenir et qu'ils faisaient moins de place à la religion en vieillissant. Un niveau plus bas de polyvictimisations était significativement associé à un plus haut niveau du sentiment d'importance, $p<, 001$, de raison d'être, $p<, 001$, et de motivation relationnelle, $p=, 01$.

Tableau 3. Moyennes et erreur-standards des forces psychosociales et du bien-être en fonction du groupe de soutien social

\begin{tabular}{|c|c|c|c|c|c|c|}
\hline & $\begin{array}{l}\text { Isolé } \\
M(E S)\end{array}$ & $\begin{array}{c}\text { Pris en charge } \\
M(E S)\end{array}$ & $\begin{array}{c}\text { Repoussé } \\
M(E S)\end{array}$ & $\begin{array}{c}\text { Interconnecté } \\
M(E S) \\
\end{array}$ & $F$ & $\eta 2$ \\
\hline \multicolumn{7}{|l|}{ Forces d'autorégulation } \\
\hline Rétablir l'affect positif &,$- 29(0,07)_{\text {a }}$ &,$- 19(0,11)_{a}$ &, $14(0,13) b$ &, $38(0,08) b$ & $13,90 * * *$ & ,09 \\
\hline Endurance psychologique &,$- 28(0,08)_{a}$ &,$- 05(0,12)_{a}$ &, $32(0,13) b$ &, $26(0,08) b$ & $9,74 * * *$ &, 06 \\
\hline Contrôle des impulsions &,$- 22(0,08)$ a &,$- 02(0,11)_{a}$ &, $07(0,13)$ a &, $26(0,08) b$ & $6,38^{* * *}$ &, 04 \\
\hline Autonomie &,$- 20(0,08)$ a &,$- 07(0,12)$ a & $, 03(0,14) a, b$ & $24(0,08)_{b}$ & $5,14^{* *}$ &, 04 \\
\hline \multicolumn{7}{|l|}{ Forces de création de sens } \\
\hline Importance &,$- 45(0,07)$ a &, $17(0,10)_{b}$ &, $17(0,12)_{b}$ &, $39(0,07)_{b}$ & $26,04^{* * *}$ &, 16 \\
\hline Raison d'être &,$- 42(0,07)$ a &,$- 03(0,11)_{b}$ &, $15(0,13) b$ &, $45(0,08) c$ & $23,47^{* * *}$ &, 14 \\
\hline Sens religieux &,$- 33(0,07)$ a &, $04(0,12) b$ &, $00(0,13) b$ &, $37(0,08) c$ & $13,72 * * *$ &, 09 \\
\hline Orientation future &,$- 31(0,07)_{a}$ &,$- 10(0,11)$ a &, $26(0,13) b$ &, $34(0,08)_{b}$ & $13,37^{* * *}$ & ,09 \\
\hline Motivation relationnelle &,$- 34(0,07)_{a}$ &, $04(0,12) b$ &, $20(0,13) b$ &, $31(0,08)_{b}$ & $12,46 * * *$ & ,08 \\
\hline \multicolumn{7}{|l|}{ Forces interpersonnelles } \\
\hline Engagement des professeurs &,$- 43(0,07)$ a &, $19(0,11)_{b}$ &, $14(0,13)_{b}$ &, $35(0,08)_{b}$ & $18,77^{* * *}$ &, 12 \\
\hline Compassion &,$- 36(0,07)_{a}$ &,$- 02(0,11)_{b}$ & $, 23(0,13) b, c$ &, $35(0,08)_{c}$ & $15,81 * * *$ &, 10 \\
\hline Climat scolaire &,$- 33(0,07)_{a}$ &, $08(0,11)_{b}$ &, $04(0,13) b$ &, $37(0,08)_{c}$ & $14,88^{* * *}$ &, 10 \\
\hline Soutien de la communauté &,$- 34(0,07)_{a}$ &, $01(0,11)_{b}$ & $, 12(0,13) b, c$ &, $35(0,08)_{c}$ & $13,52 * * *$ &, 09 \\
\hline Connexion au groupe &,$- 30(0,08)$ a &,$- 03(0,12) b$ & $24(0,13) b, c$ &, $29(0,08) c$ & $10,57 * * *$ & ,07 \\
\hline \multicolumn{7}{|l|}{ Indicateurs de bien-être } \\
\hline Bien-être subjectif &,$- 47(0,07)$ a &, $19(0,11) b$ &, $01(0,12)_{b}$ &, $45(0,07)_{c}$ & $28,22 * * *$ &, 17 \\
\hline Bien-être spirituel (non-théiste) &,$- 42(0,08)$ a &,$- 15(0,11) b$ &, $19(0,13)_{c}$ &, $49(0,08) d$ & $24,87 * * *$ &, 15 \\
\hline Bien-être spirituel (théiste) &,$- 41(0,07)$ a &, $11(0,11) b$ &, $04(0,13) b$ &, $40(0,08) c$ & $18,84^{* * *}$ &, 12 \\
\hline Bien-être familial &,$- 29(0,07)_{a}$ & $, 08(0,11) b, c$ & $-, 03(0,12) a, b$ &, $31(0,08) c$ & $11,77^{* * *}$ & ,08 \\
\hline Bien-être physique &,$- 21(0,07)_{a}$ &, $11(0,11)_{b}$ & ,03 $(0,13) a, b$ &, $17(0,08)_{b}$ & $4,55 * *$ & ,03 \\
\hline Symptômes de trauma &,$- 06(0,07)_{a}$ &, $10(0,11) \mathrm{a}$ &,$- 10(0,13)_{a}$ &, $04(0,08)_{a}$ & 0,78 &, 01 \\
\hline
\end{tabular}

Note. ${ }^{* * *} p<, 001 ;{ }^{* *} p<, 01$. Les moyennes avec des indices distincts sont significativement différentes les unes des autres, $p<, 05$. Toutes les moyennes ont été converties en scores z (moyenne $=0 ; E T=1$ ), les scores les plus élevés indiquant des niveaux plus élevés de chaque force. Les moyennes et les erreur-types de mesure sont ajustées en fonction de l'âge, du sexe et de l'historique de victimisation.

Les forces interpersonnelles. Chacune des forces interpersonnelles a montré un patron similaire à celles d'autorégulation et de création de sens, avec le groupe Isolé rapportant les niveaux les plus bas de forces interpersonnelles, le groupe Interconnecté, les plus élevés, et les groupes Repoussé et Pris en charge, se situant au milieu. Cependant, le groupe Repousséressemblait davantage au groupe Interconnectéque le groupe Pris en charge. Toutes les cinq ANCOVAs pour les forces interpersonnelles étaient significatives, $p<, 001$. Voir Tableau 3. Pour le soutien de la communauté, la compassion et la connexion au groupe, le groupe Repoussé était statistiquement similaire au groupe Interconnecté, alors que le Pris en charge, était significativement plus bas (pour les comparaisons appariées, $p$ $<, 05)$. Cela peut suggérer que les jeunes qui ne reçoivent pas de soutien social cherchent peut-être à établir des liens avec des groupes ou à développer de la compassion pour les autres afin de compenser. Pour ce qui est de l'engagement des enseignants, le groupe Isolé était significativement plus faible que les trois autres, qui étaient statistiquement similaires.

L'âge était une covariable significative pour la compassion, $p=, 01$, et le climat scolaire, $p=, 001$, avec les participants plus âgés rapportant plus de compassion et un meilleur climat scolaire que les participants plus jeunes. Le 
sexe était une covariable significative pour la compassion, $p<, 001$, les femmes rapportant plus de compassion que les hommes. La polyvictimisation était également une covariable significative pour le soutien communautaire, $p=, 01$, et le climat scolaire, $p<, 001$, les participants rapportant des scores plus élevés lorsque la victimisation était plus faible que lorsqu'elle était plus élevée.

\section{Quatre groupes de soutien social et fonctionnement actuel}

Les groupes de soutien social différaient sur cinq des six indicateurs de bien-être, $p<, 01$, à l'exception des symptômes traumatiques qui n'étaient pas significatifs. Bien que le groupe Repoussé, dans l'ensemble, ait déclaré faire mieux que le groupe Pris en charge dans les trois domaines des forces psychosociales (régulation, création de sens et relations interpersonnelles), cette tendance n'a pas été observée dans les mesures du bien-être, sauf pour le bien-être spirituel non-théiste. Bien que les deux groupes mixtes se soient à nouveau situés entre les groupes /solé et Interconnectésur toutes les mesures de fonctionnement, le groupe Pris en charge présentait, de façon générale, des scores légèrement (mais non significativement) plus élevés que le groupe Repoussé. Étonnamment, il n'y avait pas de différences significatives pour les symptômes traumatiques. Le bien-être spirituel non-théiste était la seule variable pour laquelle les quatre groupes étaient significativement différents les uns des autres. Voir le Tableau 3.

L'âge était une covariable significative pour les symptômes traumatiques, $p<, 05$, et le bien-être spirituel théiste, $p<, 01$, les participants plus âgés rapportant plus de symptômes traumatiques et moins de bien-être théiste que les participants plus jeunes. Le sexe était une covariable significative du bien-être physique, $p<, 05$, et des symptômes traumatiques, $p<, 05$, les hommes déclarant un meilleur bien-être physique que les femmes et les femmes déclarant plus de symptômes traumatiques que les hommes.

La polyvictimisation était une covariable significative pour le bien-être subjectif, le bien-être familial et le bienêtre physique (tous à $p<, 001$ ), les participants ayant déclaré des scores de bien-être plus élevés lorsque la polyvictimisation était faible que lorsque la victimisation était élevée. La polyvictimisation a également montré une association positive avec les symptômes traumatiques $(p=, 05)$ et le bien-être spirituel non-théiste $(p<, 05)$.

\section{Discussion}

À notre connaissance, cette étude est la première à explorer à la fois l'impact de la recherche et de l'obtention du soutien social chez les jeunes en utilisant une approche à double facteur. Nous avons examiné quatre groupes : les jeunes du groupe Interconnecté, qui ont obtenu un score élevé de recherche et de réception de soutien social ; les jeunes du groupe Isolé, qui ont déclaré avoir peu cherché et peu reçu de soutien ; un groupe Repoussé qui a déclaré avoir beaucoup recherché de soutien social, mais en avoir peu reçu; et un groupe Pris en charge qui a reçu beaucoup de soutien social malgré en avoir peu recherché. Ce dernier groupe, particulièrement pertinent pour les jeunes ayant des donneurs de soins, des enseignants ou d'autres adultes qui tentent de s'occuper d'eux même s'ils n'expriment pas facilement leurs besoins, a fait l'objet de peu d'attention de la part des chercheurs. Des travaux antérieurs sur le modèle à double facteur de la santé mentale ont révélé des nuances qui n'étaient pas apparentes en examinant uniquement la psychopathologie ou le bien-être comme constructions distinctes (Antaramian et al., 2010; Greenspoon \& Saklofske, 2001; Suldo \& Shaffer, 2008). De même, cette approche à double facteur du soutien social peut intégrer les résultats de travaux précédemment menés sur le soutien social et la recherche d'aide de façon séparée, et fournir des indications qui pourront mieux orienter les recherches, la prévention et les interventions futures.

Tel qu'attendu, et conformément au Resilience Portfolio Mode/ainsi qu'aux recherches antérieures (Chu et al., 2010; Grych et al., 2015), les jeunes du groupe Interconnectéont obtenu des scores significativement plus élevés que le groupe Isolé pour chaque force psychosociale et chaque indicateur de fonctionnement actuel, à l'exception des symptômes traumatiques. La différence moyenne entre ces deux groupes était d'un écart-type de 0,65 (en utilisant les scores-z). Il y avait une certaine variabilité dans les schémas de significativité de ces groupes par rapport aux deux profils mixtes, mais les groupes Repoussé et Pris en charge se situaient généralement entre les deux et étaient significativement différents des groupes Interconnecté et /solésur de nombreuses variables, y compris la raison d'être, la création de sens religieux, le climat scolaire, le bien-être subjectif, le bien-être familial et le bien-être spirituel théiste et non-théiste.

Plus particulièrement, le groupe Repoussé a rapporté des niveaux plus élevés de certaines forces psychosociales que le groupe Pris en charge, notamment quant au rétablissement de l'affect positif, l'endurance et l'orientation future. Le groupe Repoussé a également montré un bien-être spirituel non-théiste plus élevé que le 
groupe Pris en charge. Le groupe Pris en charge n'a jamais été significativement plus élevé que le groupe Repoussé. Dans l'ensemble, le groupe Repoussé était similaire au groupe Interconnectéen ce qui concerne la plupart des forces psychosociales (statistiquement indiscernable sur 10 des 14). En moyenne, le groupe Repoussé se situait à un écarttype de 0,19 en dessous du groupe Interconnecté. En revanche, le groupe Pris en charge était souvent inférieur au groupe Interconnecté (significativement plus bas sur 11 des 14 points forts), avec des scores moyens se situant à un écart-type de 0,34 en dessous du groupe Interconnecté. Cependant, bien que les différences ne soient pas statistiquement significatives, ce schéma variait pour la plupart des variables de bien-être (toutes sauf le bien-être spirituel non-théiste), le groupe Pris en charge rapportant un bien-être légèrement plus élevé que le groupe Repoussé. Les groupes Pris en charge et Repoussése situaient tous deux significativement plus bas que le groupe Interconnecté pour trois des variables de bien-être, le bien-être subjectif et les deux formes de bien-être spirituel.

Le Resilience Portfolio Mode/ reconnaît que même si les portfolios de forces plus denses et plus diversifiés sont bénéfiques, certaines personnes n'ont pas les ressources ou les moyens de les utiliser. Concernant s'il est préférable de rechercher ou de recevoir du soutien, ces résultats suggèrent que les compétences et les attitudes individuelles peuvent être plus utiles aux jeunes que les offres de soutien externes. Il existe un vieil adage en psychothérapie qui dit: « Ne travaille jamais plus fort que ton client», et il est possible qu'offrir du soutien social lorsqu'un jeune n'a pas demandé d'aide ou qu'il résiste à reconnaître son besoin d'aide soit contre-productif. Cette question peut être particulièrement importante pour les jeunes qui ont moins de contrôle sur le moment et la manière dont ils sollicitent une psychothérapie et d'autres services de soins de santé. II s'agit d'un phénomène encore peu étudié qui nécessite une plus grande attention.

Une autre possibilité est que les jeunes du groupe Repoussé « s'endurcissent », c'est-à-dire qu'ils essaient de se renforcer ou de réduire leurs vulnérabilités (Rutter, 2012) en réponse à un environnement qui offre peu de soutien, que ce soit par manque de ressources ou par négligence ou hostilité. Cela se reflète dans la nature des forces distinguant davantage les groupes Repoussé et Pris en charge. Une plus forte orientation vers l'avenir peut être due à la motivation d'échapper à un environnement dysfonctionnel, et une plus grande endurance peut indiquer que les jeunes s'endurcissent pour survivre dans des circonstances difficiles. L'hypothèse de l'endurcissement est également soutenue par les différences observées dans les mesures de bien-être : les scores élevés du groupe Repoussé aux différentes forces montraient peu de bénéfices puisque leur bien-être actuel se situait plus près du groupe Pris en charge que du groupe Interconnecté.

Ces résultats sont largement comparables à ceux d'autres publications qui ont établi une association positive entre le soutien social et le fonctionnement actuel des jeunes (Chu et al., 2010). Comme on l'a déjà noté (Chu et al., 2010), les recherches antérieures sur le soutien social, bien que cohérentes avec un effet global positif, ont également rapporté des résultats plus hétérogènes que prévu et les présents résultats suggèrent une explication à cela. Même si des recherches antérieures ont étudié différents types de soutien social (degré de soutien provenant de différentes sources, accès à différents types de ressources), peu d'entre elles ont étudié les modes de recherche et d'obtention et, à notre connaissance, aucune étude n'a examiné l'équivalent du groupe Pris en charge de la présente étude. Bien que des recherches antérieures aient étudié l'association du soutien social avec d'autres indicateurs de l'écologie sociale (Turner et al., 2017), les résultats de cette étude suggèrent que les liens entre le soutien social et d'autres forces ne se limitent pas aux indicateurs environnementaux tels que le climat scolaire ou le soutien communautaire, mais s'étendent à d'autres types de forces. Les résultats des covariables sont largement similaires à ceux des recherches précédentes, de nombreux facteurs s'améliorant avec l'âge (à l'exception des facteurs religieux qui diminuent généralement pendant cette période de développement), et des résultats plus dispersés en ce qui a trait aux différences entre les sexes (Hagler et al., 2016; Hamby et al., 2017). Le fait que la polyvictimisation soit inversement associée aux nombreuses forces et indicateurs de fonctionnement global est aussi conforme aux recherches précédentes (p. ex., Hamby, Grych, et al., 2018; Turner et al., 2017).

Une question soulevée par ces données est de savoir dans quelle mesure une tendance à la recherche de soutien social doit être considérée comme une force individuelle par rapport à un phénomène exclusivement relationnel. De nombreuses mesures et plusieurs modèles de soutien social sont axés sur les ressources externes, telles que la taille des réseaux sociaux, la diversité des ressources accessibles, la quantité de soutien fourni ou la satisfaction à l'égard du soutien fourni (Barrera, 1986; Sarason et al., 1983; Sherbourne \& Stewart, 1991; Zimet et al., 1988), et ne mettent pas l'accent sur ce que font les individus pour favoriser les relations sociales ou solliciter de l'aide. Ces données montrent que les deux sont nécessaires pour un bénéfice optimal et que les actions et les attitudes individuelles peuvent être plus importantes pour le fonctionnement des jeunes que ce qui est offert de l'extérieur. 


\section{Forces et limites de l'étude}

Les résultats de cette étude devraient être évalués en tenant compte des forces et des limites du projet. Ce dernier enrichit les études sur le soutien social en séparant la recherche de soutien social de la réception de soutien social et en se concentrant sur les jeunes qui peuvent recevoir un niveau élevé de soutien malgré un faible niveau de recherche. Le projet élargit également le nombre de caractéristiques et d'indicateurs de bien-être qui ont été étudiés en relation avec le soutien social. L'étude approfondit également les informations sur le soutien social dans les communautés à faible revenu du sud des États-Unis. Néanmoins, il serait utile de reproduire ces résultats dans d'autres populations provenant d'autres régions des États-Unis et du monde. Cette étude transversale était un moyen approprié et rentable d'explorer de nouvelles idées, mais les résultats gagneraient à être reproduits dans une étude longitudinale.

\section{Implications pour la recherche}

Ce modèle de soutien social à double facteur est une approche prometteuse pour élargir notre compréhension de la manière d'aider les jeunes qui ont connu l'adversité. Les recherches futures peuvent faire davantage pour explorer pourquoi des différences ont été constatées pour certaines formes de bien-être, comme le bien-être subjectif ou spirituel, mais pas pour le bien-être physique ou les symptômes traumatiques. II serait également utile d'examiner, dans des études futures avec des échantillons plus importants ou plus diversifiés, si le modèle à double facteur varie en fonction de l'âge, de l'identité raciale et ethnique, du sexe, de l'orientation sexuelle ou d'autres caractéristiques. Les recherches futures pourraient intégrer de multiples répondants ou d'autres sources de données et adopter différentes méthodes d'échantillonnage. II serait également utile d'étudier comment le modèle à double facteur de soutien social pourrait varier selon que la demande d'aide soit formelle ou informelle. Les recherches futures doivent faire plus que simplement examiner les différents indicateurs de soutien social ou d'écologie sociale et étudier les façons dont ces divers facteurs peuvent se combiner pour influencer le bien-être et la résilience des jeunes.

\section{Implications pour l'intervention et la prévention}

Les parents, les enseignants, les thérapeutes et les autres personnes qui travaillent avec des adolescents qui, pour une raison quelconque, ne peuvent ou ne veulent pas exprimer leurs besoins et rechercher du soutien, peuvent se demander s'il vaut mieux deviner ces besoins et essayer d'y répondre, ou s'il vaut mieux encourager les jeunes à aller vers les autres et à parler de leurs besoins. La présente étude montre qu'il existe un lien positif entre l'efficacité des compétences et des attitudes de recherche de soutien social et les indicateurs de forces psychosociales et de fonctionnement global, ce qui laisse penser que cette dernière stratégie pourrait peut-être apporter des avantages à plus long terme. Les résultats de cette étude suggèrent qu'il est plus important d'encourager la recherche d'aide et les attitudes positives envers le partage, la divulgation et l'obtention de conseils, que de se contenter de fournir un soutien aux jeunes, qu'ils le veuillent ou non. Bien entendu, il existe des obligations légales d'aider les jeunes dans certaines circonstances. Des techniques telles que l'entretien motivationnel (Arkowitz et al., 2015), qui met l'accent sur l'engagement des clients, est une approche basée sur les données probantes pouvant servir à travailler avec des jeunes qui peuvent avoir besoin d'aide (du point de vue d'un adulte), mais ne l'ont pas demandé.

Ces données indiquent également qu'il faut à la fois rechercher et recevoir un soutien social pour atteindre de plus hauts niveaux de fonctionnement, sur un large éventail de mesures. Les organismes et les communautés doivent s'assurer que lorsque les jeunes cherchent à obtenir un soutien social, ils le reçoivent, en particulier lorsque les familles et les pairs n'en offrent pas. Les approches multidisciplinaires, telles que les centres de protection de la jeunesse, sont une des approches conçues pour améliorer la qualité de la lutte contre la victimisation, mais des recherches supplémentaires sont nécessaires concernant les éléments qui améliorent le plus l'écologie sociale des jeunes (Elmquist et al., 2015). Une écologie sociale saine signifie un fonctionnement optimal des individus, des familles et des communautés.

\section{Financement}

Ce projet a été financé par le National Institute of Justice, Office of Justice Programs, U.S. Department of Justice (prix $n^{\circ}$ 2015-R2-CX-0004). Les opinions, constatations et conclusions ou recommandations exprimées dans ce manuscrit sont celles des auteur.es et ne reflètent pas nécessairement celles du National Institute of Justice. 


\section{Conflit d'intérêt}

Les auteur.es déclarent n'avoir aucun conflit d'intérêts.

\section{Références}

Abt SRBI. (2012). Second national survey children's exposure to violence (NatSCEV II): Methods report. Retrieved from https://www.bjs.gov/content/pub/pdf/natscev2_mr.pdf

Antaramian, S. P., Scott Huebner, E., Hills, K. J., \& Valois, R. F. (2010). A dual-factor model of mental health: Toward a more comprehensive understanding of youth functioning. American Journal of Orthopsychiatry, 80(4), $462-472$. https://doi.org/10.1111/j.1939-0025.2010.01049.x

Arkowitz, H., Miller, W. R., \& Rollnick, S. (2015). Motivational interviewing in the treatment of psychological problems. Guilford Press.

Banyard, V., Hamby, S., \& Grych, J. (2017). Health effects of adverse childhood events: Identifying promising protective factors at the intersection of mental and physical well-being. Child Abuse \& Neglect, 65, 88-98. https://doi.org/10.1016/j.chiabu.2017.01.011

Barrera, M. (1986). Distinctions between social support concepts, measures, and models. American Journal of Community Psychology, 14(4), 413-445. https://doi.org/10.1007/BF00922627

Barrett, B. J., \& Pierre, M. S. (2011). Variations in women's help seeking in response to intimate partner violence: Findings from a Canadian population-based study. Violence Against Women, 171), 47-70. https://doi.org/10.1177/1077801210394273

Bronfenbrenner, U. (1979). The ecology of human development. Harvard University Press.

Bundock, K., Chan, C., \& Hewitt, O. (2020). Adolescents' help-seeking behavior and intentions following adolescent dating violence: A systematic review. Trauma, Violence, \& Abuse, 21(2), 350-366. https://doi.org/10.1177/1524838018770412

Campbell, R., Wasco, S. M., Ahrens, C. E., Sefl, T., \& Barnes, H. E. (2001). Preventing the "second rape" rape survivors' experiences with community service providers. Journal of Interpersonal Violence, 16(12), $1239-1259$. https://doi.org/10.1177/088626001016012002

Centers for Disease Control and Prevention. (2000). Measuring health days: Population assessment of health-related quality of life. CDC.

Chan, W. Y., Hollingsworth, M. A., Espelage, D. L., \& Mitchell, K. J. (2016). Preventing violence in context: The importance of culture for implementing systemic change. Psychology of Violence, 6(1), 22-26. https://doi.org/10.1037/vio0000021

Chu, P. S., Saucier, D. A., \& Hafner, E. (2010). Meta-analysis of the relationships between social support and well-being in children and adolescents. Journal of Social and Clinical Psychology, 29(6), 624-645. https://doi.org/10.1521/jscp.2010.29.6.624

Clara, I. P., Cox, B. J., Enns, M. W., Murray, L. T., \& Torgrudc, L. J. (2003). Confirmatory factor analysis of the multidimensional scale of perceived social support in clinically distressed and student samples. Journal of Personality Assessment, 81(3), $265-270$. https://doi.org/10.1207/S15327752JPA8103_09

Conners-Burrow, N. A., Kramer, T. L., Sigel, B. A., Helpenstill, K., Sievers, C., \& McKelvey, L. (2013). Trauma-informed care training in a child welfare system: Moving it to the front line. Children and Youth Services Review, 35(11), $1830-1835$. https://doi.org/10.1016/j.childyouth.2013.08.013

Elmquist, J., Shorey, R. C., Febres, J., Zapor, H., Klostermann, K., Schratter, A., \& Stuart, G. L. (2015). A review of children's advocacy centers' (CACs) response to cases of child maltreatment in the United States. Aggression and Violent Behavior, 25, 26-34. https://doi.org/10.1016/j.avb.2015.07.002

Finkelhor, D., Hamby, S. L., Ormrod, R., \& Turner, H. (2005). The Juvenile Victimization Questionnaire: reliability, validity, and national norms. Child Abuse \& Neglect, 2944), 383-412. https://doi.org/10.1016/j.chiabu.2004.11.001

Finkelhor, D., Vanderminden, J., Turner, H., Shattuck, A., \& Hamby, S. (2014). Youth exposure to violence prevention programs in a national sample. Child Abuse \& Neglect, 38(4), 677-686. https://doi.org/10.1016/j.chiabu.2014.01.010

Foynes, M. M., \& Freyd, J. J. (2011). The impact of skills training on responses to the disclosure of mistreatment. Psychology of Violence, 1(1), 66-77. https://doi.org/10.1037/a0022021

Frison, E., \& Eggermont, S. (2015). The impact of daily stress on adolescents' depressed mood: The role of social support seeking through Facebook. Computers in Human Behavior, 44, 315-325. https://doi.org/10.1016/j.chb.2014.11.070

Fritz, J., de Graaff, A. M., Caisley, H., Van Harmelen, A.-L., \& Wilkinson, P. O. (2018). A systematic review of amenable resilience factors that moderate and/or mediate the relationship between childhood adversity and mental health in young people. Frontiers in Psychiatry, 9(230). https://doi.org/10.3389/fpsyt.2018.00230

Galesic, M., \& Bosnjak, M. (2009). Effects of questionnaire length on participation and indicators of response quality in a web survey. Public Opinion Quarterly, 73, 349-360. https://doi.org/10.1093/poq/nfp031 
Greenspoon, P. J., \& Saklofske, D. H. (2001). Toward an integration of subjective well-being and psychopathology. Social Indicators Research, 54(1), 81-108. https://doi.org/10.1023/A:1007219227883

Grych, J., Hamby, S., \& Banyard, V. (2015). The resilience portfolio model: Understanding healthy adaptation in victims of violence. Psychology of Violence, 5(4), 343-354. https://doi.org/10.1037/a0039671

Hagler, M., Grych, J., Banyard, V., \& Hamby, S. (2016). The ups and downs of self-regulation: Tracing the patterns of regulatory abilities from adolescence to middle adulthood in a rural sample. Journal of Rural Mental Health, 40(3-4), 164-179. https://doi.org/10.1037/rmh0000053

Hamby, S., Grych, J., \& Banyard, V. (2018). Resilience portfolios and poly-strengths: Identifying protective factors associated with thriving after adversity. Psychology of Violence, 8(2), 172-183. https://doi.org/10.1037/vio0000135

Hamby, S., Segura, A., Taylor, E., Grych, J., \& Banyard, V. (2017). Meaning making in rural appalachia: Age and gender patterns in seven measures of meaning. Journal of Happiness and Well-being, 5(2), 168-186.

Hamby, S., Taylor, E., Smith, A., \& Blount, Z. (2018). Resilience portfolio questionnaire manual: Scales for youth. Sewanee, TN: Life Paths Research Center. https://doi.org/10.13140/RG.2.2.27296.74243

Kelly, J. G. (1968). Towards an ecological conception of preventive interventions. In J. W. Carter (Ed.), Research contributions from psychology to community mental health (pp. 75-99). Behavioral Publications.

Kim, H. S., Sherman, D. K., \& Taylor, S. E. (2008). Culture and social support. American Psychologist, 63(6), 518. https://doi.org/10.1037/0003-066X

Malecki, C. K., \& Demaray, M. K. (2002). Measuring perceived social support: Development of the child and adolescent social support scale (CASSS). Psychology in the Schools, 39(1), 1-18. https://doi.org/10.1002/pits.10004

Roberts, L. T., Hamby, S., Banyard, V. L., \& Grych, J. (2015). Beyond collective efficacy: New brief measures to assess the outer layers of the social ecology. American Journal of Psychology and Behavioral Sciences, 2(2), 14-23.

Rutter, M. (2012). Resilience as a dynamic concept. Development and Psychopathology, 24(2), 335-344. https://doi.org/10.1017/S0954579412000028

Sampson, R. J., Raudenbush, S. W., \& Earls, F. (1997). Neighborhoods and violent crime: A multilevel study of collective efficacy. Science, 277(5328), 918-924. https://doi.org/10.1126/science.277.5328.918

Sarason, I. G., Levine, H. M., Basham, R. B., \& Sarason, B. R. (1983). Assessing social support: The social support questionnaire. Journal of Personality and Social Psychology, 44(1), 127-139. https://doi.org/10.1037/0022-3514.44.1.127

Sherbourne, C. D., \& Stewart, A. L. (1991). The MOS social support survey. Social Science \& Medicine, 32(6), 705-714. https://doi.org/10.1016/0277-9536(91)90150-B

Suldo, S. M., \& Shaffer, E. J. (2008). Looking beyond psychopathology: The dual-factor model of mental health in youth. School Psychology Review, 37(1), 52-68. https://doi.org/10.1080/02796015.2008.12087908

Sullivan, T. P., Schroeder, J. A., Dudley, D. N., \& Dixon, J. M. (2010). Do differing types of victimization and coping strategies influence the type of social reactions experienced by current victims of intimate partner violence? Violence Against Women, 16(6), 638657. https://doi.org/10.1177/1077801210370027

Trickett, E., Kelly, J. G., \& Vincent, T. (1983). The spirit of ecological inquiry in community research. In D. Klein \& E. Susskind (Eds.), Knowledge building in community psychology (pp. 283-333). Praeger.

Turner, H. A., Shattuck, A., Finkelhor, D., \& Hamby, S. (2017). Effects of poly-victimization on adolescent social support, self-concept, and psychological distress. Journal of Interpersonal Violence, 32(5), 755-780. https://doi.org/10.1177/0886260515586376

Zimet, G. D., Dahlem, N. W., Zimet, S. G., \& Farley, G. K. (1988). The multidimensional scale of perceived social support. Journal of Personality Assessment, 52(1), 30-41. https://doi.org/10.1207/s15327752jpa5201_2 